\title{
La bibliothèque de Guillaume Budé
}

\section{Luigi-Alberto Sanchi}

\section{(2) OpenEdition}

\section{Journals}

Édition électronique

URL : http://journals.openedition.org/aes/1273

DOI : 10.4000/aes. 1273

ISSN : 2258-093X

\section{Éditeur}

Laboratoire LISAA

\section{Référence électronique}

Luigi-Alberto Sanchi, «La bibliothèque de Guillaume Budé », Arts et Savoirs [En ligne], 10 | 2018, mis en ligne le 21 novembre 2018, consulté le 01 mai 2019. URL : http://journals.openedition.org/aes/1273 ; DOI : 10.4000/aes. 1273

Ce document a été généré automatiquement le 1 mai 2019.

Centre de recherche LISAA (Littératures SAvoirs et Arts) 


\title{
La bibliothèque de Guillaume Budé
}

\author{
Luigi-Alberto Sanchi
}

1 Il y a presque un siècle, les fondateurs de la société parisienne d'édition «Les Belles Lettres » ont choisi de placer sous le nom de Guillaume Budé (1468-1540) ${ }^{1}$ la Collection des Universités de France, créée dans le but de proposer au public cultivé francophone des éditions critiques de toute la littérature grecque et latine accompagnées de traductions fiables. La double série de cette prestigieuse collection, toujours vivante, compte aujourd'hui des centaines de volumes; elle est connue en France comme à l'international par l'appellation de "Collection Budé ». On ne saurait mieux dire : riche en manuscrits et imprimés des deux langues, la bibliothèque que Guillaume Budé a pu réunir au cours de sa vie correspond tout à fait à cette ambition universelle, comme on pourra le constater en lisant le présent abrégé, même si elle n'est pas à ce jour entièrement reconstituée ${ }^{2}$. Les études sur ses importants travaux philologiques, les Commentaires de la langue grecque (Commentarii linguce Grece) et L'As et ses fractions (De Asse et partibus eius libri $V)^{3}$ ont en effet permis de mieux cerner sa bibliothèque de travail, grâce aux citations fréquentes et très précises sur lesquelles Budé s'appuie. Car, s'il n'a pas été un collectionneur de manuscrits comme il en existera aux générations après la sienne, Budé a néanmoins cherché à se procurer le plus grand nombre possible d'auteurs anciens. Un dépouillement encore à venir de son premier chef-d'œuvre, les Annotations aux Pandectes ( Annotationes in Pandectas) $)^{4}$, ainsi que du vaste ensemble d'excerpta réunis dans les sept carnets de lieux communs qui nous restent de Budés, permettrait de parachever une liste déjà très nourrie.

2 Pour esquisser la composition de cette collection de livres, il faut d'abord rappeler qu'elle fut entamée par le père de Guillaume, Jean (dit Jean II) Budé - «librorum emacissimus » comme Guillaume le qualifie dans une lettre autobiographique ${ }^{6}$ - puisque celui-ci en a évidemment profité, du moins à ses débuts. Si les études de droit à Orléans justifient sa culture juridique et sa connaissance précise du corpus de Justinien (Institutes, Code, Pandectes), de la Glose d'Accurse et des usuels de l'époque en la matière, ses fréquentations d'humanistes le mettent bientôt en contact avec le maître de cette génération, Jacques Lefèvre d'Étaples, sans doute peu après le retour en France de celui-ci en 1492, et, par son intermédiaire, avec l'architecte et humaniste italien Giovanni 
Giocondo, qui fait partager aux auditeurs parisiens ses travaux sur Vitruve et sur Pline le Jeune. Entamé par Budé vers 1494, l'apprentissage de la langue grecque le rapproche d'abord du maître de grec et copiste Georges Hermonyme de Sparte, qui a aussi copié pour lui plusieurs manuscrits grecs de contenu classique et scripturaire ${ }^{7}$ et peut être la source de quelques lectures grecques supplémentaires, mais aussi de Janus Lascaris, illustre philologue, éditeur et diplomate qui a été ami de Budé, allant jusqu'à lui prêter une caisse de ses manuscrits grecs au contenu non précisé, et qui, résidant entre Milan et Venise, l'a aidé à acquérir des manuscrits et des éditions italiennes, comme en témoignent leurs échanges épistolaires ${ }^{8}$. Outre Lascaris, Budé a bénéficié d'au moins un autre relais dans la Cité des Doges: le jeune humaniste Lazare de Baïf, nommé ambassadeur à Venise de 1530 à 1534, lui a procuré un manuscrit grec d'Appien, comme Budé l'écrit dans le De Asse. Bien évidemment, Budé s'est aussi procuré les volumes de plusieurs auteurs latins, surtout Pline l'Ancien, central pour ses recherches dans le De Asse , dont il a étudié trois différents manuscrits, désormais identifiés, et au moins deux éditions imprimées.

3 Mis à part ces cas bien documentés, il est aisé de montrer que Budé a été un grand acheteur de livres imprimés en Italie, en particulier de princeps grecques issues des presses aldines, mais aussi d'incunables d'auteurs latins classiques et patristiques, ou de versions latines humanistes d'œuvres grecques: ce sont là les bases de ses recherches linguistiques, philologiques et historiques sur l'Antiquité, sans oublier les "usuels » grammaticaux latins et grecs antiques ou médiévaux (Hésychius, Pollux, la Souda, les Étymologies d'Isidore de Séville ou les Derivationes d'Uguccione de Pise), en partie déjà identifiés, et les commentaires et encyclopédies qu'ont produits les humanistes du Quattrocento, en premier lieu les Castigationes Pliniance et in Pomponium Melam d'Ermolao Barbaro, la Roma Triumphans de Biondo Flavio, le Cornu Copice de Niccolò Perotti, les Élégances de Laurent Valla et les Miscellanées d'Ange Politien, mais aussi les annotations de divers humanistes à des auteurs comme Juvénal, Suétone, Martial ou Valère Maxime.

Guillaume Budé a donc été un lecteur vorace et tentaculaire qui, de plus, n'a pas cessé de revenir à ses classiques en préparant les nouvelles éditions de ses ouvrages érudits, comme le montrent les couches textuelles successives dont on peut faire état pour les Commentaires de la langue grecque (1529 et 1548 , posthume) ${ }^{9}$ et surtout pour le De Asse: $1515,1516,1522,1524,1527,1532$ et 1541 , posthume ${ }^{10}$. Dans la tentative de réunir les données acquises à ce jour, à notre connaissance, nous donnons ci-après le tableau des quelque deux cents volumes que Budé a possédés ou probablement utilisés, offrant un aperçu rapide de cette grande variété et du sérieux qui a caractérisé les travaux du grand humaniste parisien ; in fine, un index des auteurs.

\section{Livres de Guillaume Budé ou étudiés par lui}

Le présent tableau est articulé en huit sections, dont les six premières font alterner les domaines grec et latin, consacrées aux ensembles suivants : ouvrages médiévaux de grammaire et de lexicologie (I et II), œuvres classiques ou tardives en vers (III et $\mathrm{IV})^{11}$ et en prose (V et VI), Écriture sainte et textes patristiques (VII) et littérature et érudition du $\mathrm{VII}^{\mathrm{e}}$ siècle au Quattrocento (VIII). Un astérisque précède les quarante-six pièces identifiées à notre connaissance ; pour les textes de la bibliothèque de travail de Budé, reconstituée à travers les seules citations, les ouvrages érudits de Budé dont 
elles émanent sont donnés en sigles ${ }^{12}$ : Ann(otationes in XXIV Pandectarum libros), D (e) A(sse et partibus eius libri V) ${ }^{13}$, Comm(entarii linguæ Græcæ), Carnets (carnets autographes, numérotés de I à VII) ${ }^{14}$.

\section{Ouvrages de grammaire et lexicologie grecques}

1. Apollonius Dyscole, Syntaxe : Comm.

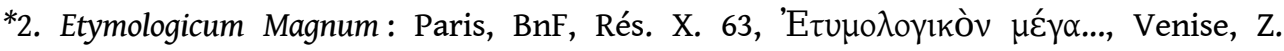
Calliergis pour N. Vlastos, 1499, avec de fréquentes annotations de Budé, renvoyant notamment à Eustathe ${ }^{15}$.

*3. [Glossaires] : ms. Wien, ÖnB, Phil. gr. 285, copié par Hermonyme et annoté par celui-ci et par Budé; contient plusieurs glossaires élémentaires, des gloses grammaticales, un lexique homérique, un opuscule de Manuel Moschopulus et un fragment de sa Métrique; les f. 1-39 manquent.

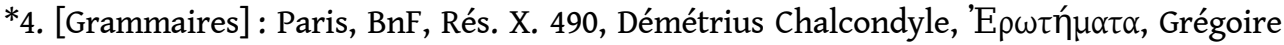

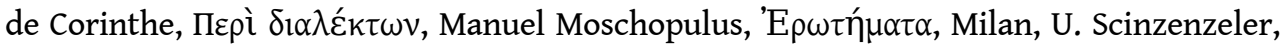
c. 1493 , avec d'abondantes annotations de Budé ${ }^{16}$.

5. Harpocration, Lexique des dix orateurs : DA, Comm.

6. Hésychius, Lexique: DA, Comm.

*7. Magister, Thomas, Choix de substantifs et verbes attiques : ms. Leyde, Univ. Bibl., Voss. gr., Fol. 71, copié par Hermonyme, qui attribue à Moschopulus la paternité de l'opuscule ; ajouts et corrections de Budé.

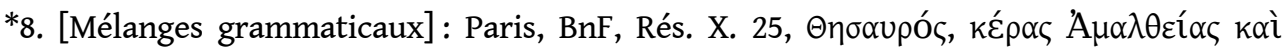

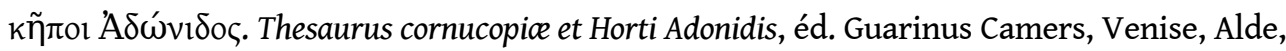
1496. Volume réunissant, outre des listes, index et tableaux de conjugaison, plusieurs

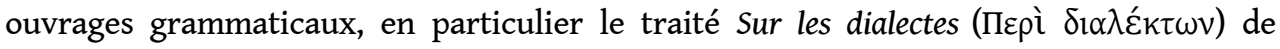
Grégoire de Corinthe, que Budé a abondamment annoté s'agissant de l'ionien ${ }^{17}$.

*9. [Opuscules grammaticaux]: ms. Turin, Bibl. Naz. Univ., IV. B. III. 9, copié par Hermonyme et entièrement annoté par Budé ; contient un glossaire anonyme De verborum significatione, des extraits du De figuris poeticis de Georges Chœroboscus, la De dictionum affectionibus de Manuel Moschopulus et le Choix de vocables attiques de Thomas Magister.

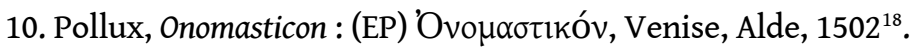

*11. Pollux (Ps.-), De cottidiana locutione, et épîtres diverses (gr., lat.) : ms. Paris, BnF, gr. 3049, copié par Hermonyme.

12. Souda [Suidas], Lexique : prob. (EP) Milan, 1499 ; DA, Comm.

\section{Ouvrages de grammaire et lexicologie latines}

13. Diomède, Ars Grammatica : Comm.

14. Priscien, œeuvre, incluant l'opuscule De figuris numerorum et le Carmen de ponderibus et mensuris attribué à Remius Favinus (nom incertain) : au moins un imprimé ${ }^{19} ; D A$.

15. Nonius Marcellus, De compendiosa doctrina : DA, Comm.

16. Uguccione de Pise, Derivationes : DA.

17. Valerius Probus, Notarum laterculi ${ }^{20}: D A$.

\section{Littérature grecque : œuvres en vers}

18. Anthologie grecque : Comm. (« in Epigrammatis »). 
19. Aristophane (et scholies) : prob. (EP) Venise, Alde, 1498 ; DA, Comm.

20. Ésope, Fables et autres auteurs : prob. Vita et Fabellæ Æsopi..., Venise, Alde, 1505; cette édition contenant, entre autres, le De natura deorum de Lucius Annæus Cornutus et 21. Les Hiéroglyphes d'Horapollon permettrait d'en expliquer les citations, respectivement dans Comm. et DA.

21. Euripide, Tragédies avec scholies : prob. éd. Venise, Alde, 1503 ; Comm.

*22. Homère, Iliade, Odyssée et scholies et corpus homérique: (EP) Princeton, Firestone

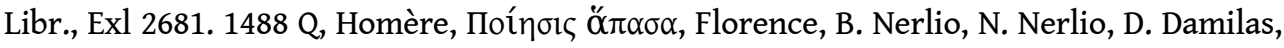
1488, t. I-II (I. : Ps.-Hérodote, Vie d'Homère ; Ps.-Plutarque, Homère ; Dion Chrysostome, Disc. sur Homère; Iliade et scholies. II.: Odyssée et scholies, Batrachomyomachie, Hymnes), richement annoté par Budé, qui semble avoir eu accès aux scholies contenues dans une source proche du ms. Venetus A d'Homère (Venise, Bibl. St-Marc, gr. 454), sans doute obtenue par l'intermédiaire de Lascaris ${ }^{21}$; voir aussi infra, n. 89.

*23. Hésiode, Théogonie, Les Travaux et les Jours : voir infra, Théocrite, n. 25.

24. Sophocle, Tragédies avec scholies : prob. (EP) Venise, Alde, 1502 ; Comm.

*25. Théocrite, Idylles: (EP) Tours, Bibl. Munic., Rés. 7575, Theocriti Eclogæ XXX..., Venise, Alde, 1495 ; comprend également les poèmes d'Hésiode, les Sentences élégiaques de Théognis, les Vers dorés de Pythagore et d'autres recueils de formes brèves.

*26. Théognis, Poèmes élégiaques : voir supra, Théocrite, n. 25.

\section{Littérature latine : œuvres en vers}

27. Catulle, Poèmes : DA, Comm.

28. Horace, Épitres, Odes, Satires : DA, Comm.

39. Juvénal, Satires : un exemplaire avec les annotations de Domizio Calderini et de Giorgio Merula $^{22}$; Ann., DA, Comm.

30. Lucain, Pharsale (La Guerre civile): DA, Comm.

31. Lucrèce, La Nature : Comm.

32. Martial, Épigrammes : DA, Comm.

33. Ovide, Fastes et Héroïdes : Comm.

34. Perse, Satires : DA, Comm.

35. Plaute, Comédies : Ann., DA, Comm.

36. Térence, Comédies : Ann., DA, Comm.

37. Virgile, Bucoliques, Énéide, Géorgiques : Ann., DA, Comm.

\section{Littérature grecque : œuvres en prose}

*38. Appien : ms. Paris, BnF, gr. 1682, apporté de Venise par Lazare de Baïf, annoté par les mains de Baïf et de Budé ; comprend la Préface, le résumé du livre IV, les livres VIII, XI-XII et XIII-XVII ${ }^{23}$.

39. Appien en trad. lat. de Pier Candido Decembrio : prob. De Bellis ciuilibus romanis, ex Appiano Alexandrino traductis in Latinum, Venise, Vindelinus de Spire, 1472 (diverses éd.); DA.

*40. Aristote et corpus aristotélicien : (EP) Leyde, Bibl. der Rijksuniv., KL 758 A 6, Aristote, Opera, t. I, Venise, Alde, 1495; Budé avait également les quatre autres tomes de cette édition $^{24}$. Dans les t. I, II et IV (datés 1497), elle contient en outre les écrits de plusieurs autres auteurs, que Budé cite également: Porphyre, Isagoge (t. I); Diogène Laërce, Vie d'Aristote, Vie de Théophraste, Jean Philopon, Vie d'Aristote, Galien, De philosopho historia, 
Philon, De mundo, ${ }^{25}$ Théophraste, Le feu, Les vents, Les Pierres (t. II) ; Id., Recherches sur les plantes, Causes des plantes, Métaphysique, Alexandre d'Aphrodise, Problèmes (t. IV) ; voir aussi infra, n. 192.

41. Aristote, "Dialectique » : sans doute un ms. grec de Lascaris, mentionné sous ce titre dans une lettre à celui-ci ${ }^{26}$.

42. Aristote, Éthique à Nicomaque, trad. lat. de Jean Argyropoulos : Comm.

43. Arrien, Histoire d'Alexandre : prob. (EP) Venise, Zanetti et Trincavelli, 1535 ; DA, Comm. 44. Arrien, trad. lat. de Bartolomeo Fazio : prob. Arriani De rebus gestis Alexandri... Latinitati donauit B. Facius, Pesaro, G. Soncino, $1508^{27}$.

45. Artémidore de Daldis, Onirocritica : prob. EP, Venise, F. d'Asola, 1518 ; DA, Comm.

*46. Athénée, Les Deipnosophistes: (EP) Oxford, Bodl. Libr., Auct. 1R 1. 9, A日qvaíov

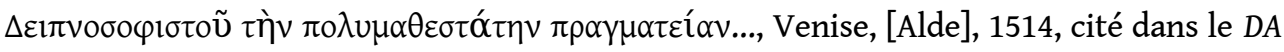
dès la première édition de $1515^{28}$.

47. Cebetis Tabula : Comm.

*48. Démosthène, Discours : (EP) Berlin, Stadtbibl. Preuss. Kulturbesitz, Haus 2, libri impr. cum notis manuscr., 7, Orationes duce et sexaginta, Venise, Alde, 1504, avec de nombreuses annotations de Budé ; contient en outre les Hypothèses de Libanios et les Vies de Démosthène de Libanios et de Plutarque.

*49. Démosthène, Sur la couronne, Eschine, Contre Ctésiphon: ms. Paris, BnF, gr. 3004, copié par Hermonyme.

*50. Diodore de Sicile, Bibliothèque historique, l. XVII : ms. Paris, BnF, gr. 1668, copie effectuée pour le roi à la demande de Budé, annotée par ce dernier ${ }^{29}$.

51. Diogène Laërce, Vies des philosophes : prob. ms. Vatican, B.A.V., Urbin. gr. 109, ayant appartenu à Hermonyme, copié et annoté de sa main ; Ann., DA, Comm. et Carnet VI (l'EP est de 1533).

*52. Diogénien, Proverbes : ms. Oxford, Bodleian Libr., Laud. gr. 7, copié par Hermonyme ; fréquentes manicules de Budé.

53. Dioscoride, De materia medica et Ps.-Dioscorides, De venenis : prob. (EP) Venise, Alde, 1499 ; le DA, p. 570, évoque en effet un « imprimé »; Ann., DA, Comm., Carnets I-II

54. Élien, Tactique: un ms. dont l'usage est attesté dans des trad. lat. imprimées ${ }^{30}$; quelques citations dans Comm.

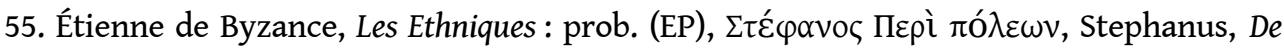
urbibus, Venise, Alde, $1502^{31}$

56. Euclide, Géométrie : Ann., Comm.

57. Flavius Josèphe, Les Antiquités juives, Contre Apion, La Guerre des Juifs : Budé a sans doute eu accès à des mss (EP Bâle, Froben, 1544); il évoque une « editio Grceca » ${ }^{32}$.

58. Galien, œuvres : prob. Opera, Venise, A. Torresano et fils, 1525 ; Comm.

59. Galien, Thérapeutique : prob. (EP), Florence, Z. Calliergès, 1500 ; Ann., Comm. ${ }^{33 .}$

60. Hérodien, Histoires : prob. (EP), Venise, Alde, 1503 ; Comm.

*61. Hérodote, Histoire: (EP) Paris, Arsenal, Fol-H-721, Herodoti libri novem, Venise, Alde, $1502^{34}$

*62. Hiéroclès, Commentaire sur les Vers dorés des pythagoriciens (extraits), Georges Hamartolos, Chronique (extraits), Exhortations morales des trois hiérarques (Grégoire de Nazianze, Basile de Césarée et Jean Chrysostome) : ms. Genève, Bibl. Publique et Univ., gr. 41, copié par Hermonyme, annoté par Budé.

63. Jean Philopon, Contra Proclum et Commentaires à Aristote: Ann., Comm.

64. Julien, Épîtres : Comm.

65. Libanios, Déclamations : prob. (EP) Ferrare, Mazocchi, 1517 ; Comm. 
66. Lucien, œuvres : prob. (EP) Florence, L. de Alopa, 1496 ; Ann., Comm.

*67. [Mélanges d'épîtres] : ms. Cambridge, Univ. Libr., Kk. VI. 23, copié par Hermonyme ; lettres de Philostrate, de Théophylacte Simocatta, du Ps.-Eschine, avec le Fragmentum Prodici (Xénophon, Mém., II, 1, 21-28) et les Characteres epistolici du Ps.-Libanios.

68. [Mélanges d'épîtres]: prob. Epistolarum Grcecarum collectio, Venise, Alde, 1499, réunissant nombre d'auteurs et de pseudépigraphes; dans les Comm., Budé cite à plusieurs reprises de courts extraits des lettres attribuées à Chion, Cratès de Mallos, Diogène de Sinope, Héraclite, Phalaride, Eschine, Démosthène, Euripide, outre celles de Platon, Alciphron, Basile de Césarée, Libanios ou Théophylacte ${ }^{35}$.

69. [Mélanges de rhétorique]: prob. Rhetores Grceci, Venise, Alde, 1508-1509, t. I-II, donnant la Rhétorique et la Poétique d'Aristote, les quatre traités d'Hermogène et les Progymnasmata d'Aphtonius, souvent cités dans les Comm., mais aussi le De interpretatione de Démétrius de Phalère et le traité de Denys d'Halicarnasse, présents dans les Comm., et d'autres opuscules rhétoriques ${ }^{36}$.

70. Paul d'Égine, Abrégé de médecine : prob. (EP) Venise, héritiers d'Alde, 1528 ; Comm.

71. Paul d'Égine, Abrégé de médecine en trad. lat. : Ann., DA.

72. Pausanias, Description de la Grèce : prob. (EP) Venise, A. Torresano, 1516 ; Comm.

73. Philostrate, Vie d'Apollonius : Ann.

74. Philostrate, Imagines : Comm.

*75. Phocylide (Ps.-), Sentences : ms. Paris, BnF, lat. 16707, textes grec et latin interlinéaire, copié par Hermonyme et acquis par Budé pour son apprentissage.

*76. Platon, Les lois avec Ps.-Platon, Minos et Épinomis : ms. Leyde, Univ. Bibl., Voss. gr., Fol. 74, copié par Hermonyme sur le ms. Paris, BnF, gr. 1807, procuré par Janus Lascaris.

77. Platon Dialogues : prob. (EP), Venise, Alde, 1513 ; DA, Comm. ${ }^{37}$.

*78. Plutarque (et Ps.-Plutarque), CEuvres morales : ms. Leyde, Univ. Bibl., Voss. gr., Quarto, 2, copié par Hermonyme et contenant vingt-et-un traités ${ }^{38}$.

79. Plutarque (et Ps.-Plutarque), CEuvres morales: (EP) Plutarchi Opuscula, Venise, Alde, 1509, acquisition attestée dans une lettre à Lascaris ${ }^{39}$.

80. Plutarque, Vies parallèles : (EP) Florence, Giunta, 1517, usage attesté dans DA, Comm., et décrit dans le $D A$ à partir de 1522 .

81. Plutarque, Vies en trad. lat. : $D A^{40}$.

82. Porphyre, Homericce Qucestiones : prob. (EP) Rome, Gymnasium Mediceum, 1518 ; Comm.

83. [Recueil d'orateurs] : prob. Rhetorum Grocorum Orationes, Venise, Alde, 1513, donnant les discours d'Eschine, Lysias, Démade (partie I), Andocide, Isée, Dinarque, Antiphon, Lycurgue, Lesbonax, Hérode Attique (partie II, avec les Vies d'Eschine et de Lysias), Isocrate, Ælius Aristide (partie III), Alcidamas (I et III), Gorgias (II et III); DA, Comm.

*84. [Recueil gnomique]: ms. Leyde, Univ. Bibl., Voss. gr., Quarto, 68, copié par Hermonyme.

85. Polybe, Histoires : Ann., DA, Comm. ; voir aussi infra, n. 97.

86. Proclus, La Sphère et Commentaire à Euclide : Comm.

87. Ptolémée, Géographie : Comm.

88. Scholies à Démosthène: DA, Comm.

89. Scholies à l'Odyssée : Didymi antiquissimi auctoris interpretatio in Odysseam, Venise, héritiers d'Alde, 1528, ou Didymi... interpretatio in Odysseam, Paris, G. Morrhy, $1530^{41}$.

90. Strabon, Géographie : sans doute un ms. $\operatorname{grec}^{42} ; D A$, Comm.

91. Strabon, Géographie, trad. lat. de Guérin de Vérone et Grégoire Tiphernas: DA (première éd. Rome, c. 1469). 


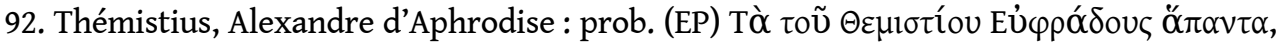

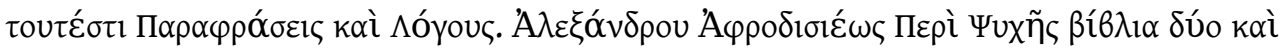

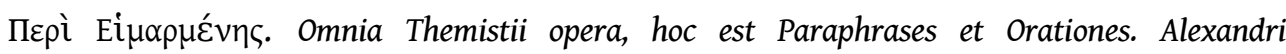
Aphrodisiensis libri duo de anima et de fato unus, Venise, héritiers d'Alde, 1534 : Budé se sert de ces auteurs dans le DA (ajouts présents seulement dans l'éd. de 1541) comme dans la deuxième édition des Comm., ce qui correspond au terminus post quem posé par cette édition.

93. Théophraste, Les Caractères : prob. (EP) Nuremberg, W. Pirckheimer, 1527 (quinze chapitres); Comm.

*94. Thucydide, La Guerre du Peloponnèse et autres auteurs grecs : ms. Paris, BnF, gr. 1638, copié par Hermonyme et annoté par Budé ; in fine, il contient aussi Lucien, Hérodote et Pro lapsu ; Maxime Planude, Éloge de l'hiver ; Démosthène, Olynthiennes I-II.

95. Xénophon, Anabase : prob. (EP) Opera, Florence, F. Giunta, 1516 ; DA, Comm.

96. Xénophon, Helléniques : prob. (EP) Venise, Alde, 1503 ; DA, Comm.

*97. Xénophon, Mémorables, Économique, Banquet et opuscules; Polybe, extrait Sur les formes de l'État: ms. Paris, BnF, gr. 1643, copié par Aristobule Apostolidis ${ }_{\mathrm{xv}}{ }^{\mathrm{e}}$ siècle) à partir d'un ms. de Janus Lascaris ; contient aussi le De equestri disciplina, le De re equestri, le De republica Lacedoemoniorum et Hiéron.

\section{Littérature latine : œuvres en prose}

98. Apulée, Métamorphoses : Ann., DA, Comm.

99. Asconius (et Ps.-Asconius), Commentaires à Cicéron : DA, Comm.

100. Aulu-Gelle, Les Nuits attiques : une édition imprimée (voir DA, p. 626-627 : « in libris impressis »).

101. Ausone, Lettres : Comm.

102. Boèce, Institution arithmétique : Comm.

103. Caton, De l'Agriculture : prob. un ms. et un imprimé ; voir DA, p. 601 (« in antiquo libro legitur ») et p. 602 (mention de l'édition imprimée éditée par G. Giocondo) ; Ann., DA, Comm.

*104. Celse, De la médecine : Paris, BnF, Rés., Fol. $\mathrm{T}^{28} 4 \alpha$, De medicina, Venise, F. Pincio pour B. Fontana, $1497^{43}$.

105. César et Ps.-César, La Guerre des Gaules, Guerre d'Afrique : Ann., DA, Comm.

106. Cicéron, œuvres ${ }^{44}$ : éditions imprimées; au moins un ms. (de St.-Victor ${ }^{45}$ ); voir aussi infra, $\mathrm{n}^{\text {os }}$ 190-191; Ann., DA, Comm.

107. Columelle, L'Agriculture (et Les arbres) : Ann., DA, Comm.

108. Cornélius Nepos, Vie d'Atticus : DA.

109. Corpus juridique de Justinien (Institutes, Code, Digeste, Novelles) avec Glose d'Accurse : au moins un ms., évoqué dans Ann. ${ }^{46}$.

110. Donat, Commentaire à Térence : DA, Comm.

111. « Donatus auctus», Vie de Virgile : $\mathrm{DA}^{47}$.

112. Eutrope, Abrégé d'histoire romaine : DA.

113. Festus / Paul Diacre, Abrégé de Verrius Flaccus : un imprimé et un ms. ${ }^{48}$; DA, Comm.

114. Firmicus Maternus, Mathesis : DA.

115. Florus, Abrégé de l'histoire romaine : DA.

116. Histoire Auguste : Ann., DA., Comm.

117. Justin, Abrégé de Trogue Pompée : DA, Comm.

118. Macrobe, Commentaire au songe de Scipion et Saturnales : DA, Comm. 
*119. Martianus Capella, Les noces de Philologie et de Mercure : Paris, BnF, Rés. Z. 3, Opus Martiani Capelle De nuptiis Philologie et Mercurii, Vicence, E. di Ca' Zeno, 1499, annoté par Budé.

120. Orose, Histoires (Contre les payens) : DA.

*121. Pline l'Ancien, Histoire naturelle ${ }^{49}$ : ms. Paris, BnF, lat. 6797 (d), ${ }_{\text {xiii }}{ }^{\mathrm{e}} \mathrm{s}$.

*122. Pline l'Ancien, Histoire naturelle : ms. Paris, BnF, lat. 6801 (h), ${ }_{\text {xiv }}{ }^{\text {e }}$ s.

*123. Pline l'Ancien, Histoire naturelle : ms. Paris, BnF, lat. 6795 (E), ${ }_{\text {ix }}{ }^{e}$ s., ou une copie.

124. Pline l'Ancien, Histoire naturelle : une éd. de Philippe Béroalde.

125. Pline l'Ancien, Histoire naturelle : une éd. de Giovanni Andrea Bussi.

*126. Pline le Jeune, Lettres : Oxford, Bodl., Auct. lat. 4, 3, deux imprimés reliés ensemble (Epistolæ, éd. Philippe Béroalde, Bologne, B. Faelli, 1498, et Ad Traianum Epistolæ..., éd. Girolamo Avanzi, [Venise], G. Taccuino, 1502), annotés par Budé d'après les cours de Giovannii Giocondo.

127. Pomponius Mela, Chorographie : DA.

128. Quinte Curce, Histoires : au moins un ms. sur parchemin et une édition imprimée $e^{50}$; DA, Comm.

129. Quintilien, Institution oratoire : Ann., DA, Comm.

130. Salluste, La Conjuration de Catilina: une éd. avec annotations de Laurent Valla (première éd. : In hoc... Bellum Catilinarium cum commento Leurentii Vallensis..., s.l.n.d., c. 1470) ; DA, Comm.

131. Salluste (Ps.-) et Cicéron (Ps.-), Invectives : DA.

132. Sénèque, Discours, Lettres à Lucilius : DA, Comm.

133. Servius, Commentaire à Virgile : Comm.

134. Suétone, Vies des douze Césars : une édition imprimée (et aucun ms.) ${ }^{51}$.

135. Tacite, Annales et Histoires : Ann., DA, Comm.

136. Tite-Live, Histoire romaine et Periochæ : Ann., DA, Comm.

137. Valère Maxime, Faits et dits mémorables : plus d'une source ${ }^{52}$.

138. Valère Maxime, Faits et dits mémorables : une éd. imprimée avec le Commentaire d'Ognibene Bonisoli (dit Leoniceno) ${ }^{53}$ : DA, Comm.

139. Varron, Économie rurale : sans doute un $\mathrm{ms}^{54}$.

140. Varron, Économie rurale : prob. (EP) par G. Merula, Venise, 1472, avec des leçons corrigées par Politien ${ }^{55}$.

141. Varron, La Langue latine : Ann., DA, Comm.

142. Végèce, Epitoma institutionum rei militaris : prob. un ms. au moins ${ }^{56}$.

*143. Vitruve, L'Architecture, Frontin, Les Acqueducs et autres ouvrages : BnF, Rés. V 318, L. Vitruuii Pollionis De architectura libri decem; Sexti Julii Frontini De aquæductibus liber unus, Venise, S. Bevilacqua, 1497, annoté par Budé ; contient en outre Cléonidès, Harmonicum introductorium et Ange Politien, Panepistemon et Lamia (exemplaire numérisé).

\section{Écriture sainte et textes patristiques}

144. Ambroise, De bono mortis : Comm.

145. Augustin, La Cité de Dieu : DA, Comm.

146. Basile de Césarée, œuvres : prob. En, amice lector, thesaurum damus..., éd. Érasme, Bâle, Froben, 1532, comprenant aussi des lettres de Grégoire de Nazianze ; Comm. (ajouts parus dans la deuxième éd. $)^{57}$.

147. Cyprien, De Dominica Oratione : DA, Comm. 
148. Cyrille d'Alexandrie : Comm.

149. Eusèbe de Césarée, Histoire ecclésiastique : DA, Comm.

150. Eusèbe de Césarée, Préparation évangélique en trad. latine : $D A, C o m m .{ }^{58}$

*151. Grégoire de Nazianze, Homélies: (EP) Tours, CESR, SR 14A/6725, Orationes lectissimce

XVI, Venise, A. Torresano et fils, 1516.

152. Grégoire de Nazianze, Poèmes : DA, Comm.

153. Grégoire de Nysse, La Création de l'homme : Comm.

154. Hilaire de Poitiers, Commentaire à l'Évangile de Matthieu : Comm.

155. Historia ecclesiastica tripertita : Comm.

*156. Jean Chrysostome, Commentaire aux Épîtres de Paul : (EP) Paris, BnF, Résac. C. 201,

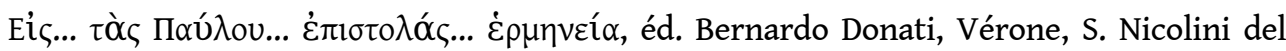
Sabbio, 1529, t. [I-]IV ${ }^{59}$.

157. Jean Chrysostome, De fato, De sacerdotio et d'autres écrits : Comm., Carnets IV et VI

158. Jean Damascène, œuvres : Comm.

159. Jérôme, Commentaires bibliques (Exod., Ezéch.), Correspondance; trad. lat. d'Eusèbe, Chronique : DA, Comm.

160. Justin (Ps.-), Exhortation aux Grecs : DA, Comm.

161. Lactance, Institutions divines : Ann., DA, Comm.

*162. Nouveau Testament grec (Évangiles) : ms. Cambridge, Univ. Libr., Ll. II. 13 (olim 2161), copié par Hermonyme.

163. Nouveau Testament grec (Évangiles) : prob. ms. Paris, BnF, Suppl. gr. 185, provenant de l'Abbaye de Saint-Victor, où Budé déclare avoir consulté un évangéliaire " très ancien " ${ }^{60}$ et où le catalogue de 1514 ne cite que ce volume ${ }^{61}$.

*164-169. Nouveau Testament grec, six mss résultant du dépeçage d'un volume copié par Hermonyme $^{62}$, annoté par Budé : Paris, BnF, gr. 59 (Actes, Jacques, Pierre I-II, Jean I-III, Judas, Apocalypse et extraits des Commentaires à l'Apocalypse d'CEcumenius et d'Aréthas [André] de Césarée), gr. 108, gr. 109, gr. 110, gr. 111 (Épîtres de Paul) ; Vatican, Reg. gr., 76 (trois Épîtres catholiques).

170. Nouveau Testament (grec et trad. lat.) d'Érasme : Novum Instrumentum omne..., Bâle, Froben, 1516, dont témoigne la correspondance de Budé avec Érasme ${ }^{63}$.

171. Origène, De principiis : prob. (EP) Venise, L. de Soardis, 1513 ; Comm. (ajouts après 1529).

*172. Pseudo-Denys l'Aréopagite, Euvres (avec scholies) : ms. Paris, BnF, gr. 447, annoté par Budé.

173. Rufin, Contre Jérôme et trad. lat. d'Origène, Homélies ${ }^{64}: D A$.

174. Septante : Ann., DA, Comm., Carnet VI.

175. Tertullien, Apologétique, De prcescriptione høereticorum : DA.

176. Théodoret, Histoire ecclésistique et Thérapeutique des maladies helléniques: Comm.

*177. Vulgate : Manchester, Chetham's Library, MUN A.9.17-20, Epistola beati Hieronimi presbiteri..., [Strasbourg, A. Rusch pour A. Koberger, Nuremberg], [avant 1480], t. I-IV, avec Glose de Nicolas de Lyre, annoté entre autres par Budé.

\section{Littérature et érudition du Moyen Âge (VII ${ }^{e}$ siècle) au Quattrocento ${ }^{65}$}

178. Barbaro, Ermolao, Castigationes Pliniance, 1493-1494 : extensivement utilisé dans le DA. 179. Béroalde (L'Ancien), Philippe, Sabellico, Marcantonio, Commentaire à Suétone : $D A^{66}$. 180. Bessarion, In Calumniatorem Platonis : Ann., Comm., Carnets V et VII. 181. Biondo, Flavio, Roma triumphans (De Roma triumphante libri X) : DA. 
182. Boèce (Ps.-), Géométrie : DA.

183. Calderini, Domizio, Annotations à Martial : $D A^{67}$.

184. Décrétales : une cit. de Grégoire X et une de Boniface VIII dans les Comm.

185. Érasme, Adages et Ciceronianus : Ann., DA, Comm. ${ }^{68}$.

186. Eustathe, Commentaires à l'Iliade et à l'Odyssée : Comm. ${ }^{69}$

187. Filetico, Martino, Commentaire aux Lettres à des familiers : $D A^{70}$.

188. Gaza, Théodore, De mensibus : DA, Comm. ${ }^{71}$.

189. Gaza, Théodore, Grammaire : Comm.

190. Gaza, Théodore, trad. grecque de Cicéron, Caton l'Ancien: prob. ms. Paris, BnF, gr. 2071, copié par Hermonyme ${ }^{72}$ [première éd. Venise, Alde, et Florence, Giunta, 1517] ; DA, Comm.

191. Gaza, Théodore, trad. grecque de Cicéron, Lalius : Comm.

192. Gaza, Théodore, trad. lat. d'Aristote, Histoire des animaux : DA, Comm. ${ }^{73}$.

193. Gaza, Théodore, trad. lat. d'Élien : Comm.

194. Gratien, Décret : Comm.

195. Hippatrica ${ }^{74}$ : Comm.

196. Isidore, Étymologies : DA, Comm.

*197. Landino, Cristoforo, Disputationes Camaldulenses : Paris, BnF, Rés. Z. 1059, Florence, N. di Lorenzo, [c. 1480].

198. Léon Magentinos, Michel Psellos et Ammonius, Commentaires à Aristote: prob. (EP) Ammonii Hermei Commentaria... Margentini [sic]..., Venise, Alde, $1503^{75}$; Comm.

199. Perotti, Niccolò, Cornu Copice : DA.

*200. Pléthon, Georges Gémiste, Epinomis, extraits des Lois (prières et hymnes) et autres écrits, ms. Vatican, BAV, Reg. gr. 184, copié par Hermonyme et annoté par Budé ; contient en outre des extraits de la Chronique d'Hamartolos, des Patria Konstantinopouleos du Ps.Codinus, d'une trad. grecque anonyme de la Rhetorica ad Herennium.

201. Politien, Ange : prob. Omnia opera, Venise, Alde, 1498 ; $D A^{76}$.

202. Raban Maur, Commentaire à l'Exode : DA.

203. Scholarios, Georges, La Trinité : Comm., carnet VI ${ }^{77}$.

204. Valla, Laurent, trad. lat. d'Hérodote : DA.

205. Valla, Laurent, Les Élégances de la langue latine : Comm. ${ }^{78}$.

206. Valturio, Roberto, La Discipline militaire (De Re militari, 1455 ; première éd. Vérone, 1472) : DA

\section{ANNEXES}

*207. Varthema, Ludovico di, Voyage: Paris, BnF, Résac. $4^{\circ} \mathrm{O}^{3} \mathrm{c} .1$, Novum Itinerarium Æthiopice, Ægypti, utriusque Arabice, Persidis, Syrice ac Indice intra et extra Gangem, Milan, G. G., B. et G. A. da Legnano, 1511. 


\section{Index des auteurs ${ }^{79}$}

ACCURSE : n. 109

ÆLIUS ARISTIDE : n. 83

ALCIDAMAS : n. 83

ALCIPHRON : n. 68

ALEXANDRE D'APHRODISE : n. 40, n. 92

AMBROISE : n. 144

AMMONIUS : n. 198

ANDOCIDE : n. 83

ANTHOLOGIE GRECQUE : n. 18

ANTIPHON : n. 83

APHTHONIUS : n. 69

APOLLONIUS DYSCOLE : n. 1

APOSTOLIDIS, ARISTOBULE : n. 97

APPIEN : n. 38, 39

APULÉE : n. 98

ARÉTHAS DE CÉSARÉE : n. 164

ARGYROPOULOS, JEAN : n. 42

ARISTOPHANE : n. 19

ARISTOTE (PS.-) : n. 40

ARISTOTE : n. 40, 42, 68, 69

ARRIEN : n. 43, 44

ARTÉMIDORE DE DALDIS : n. 45

ASCONIUS (ET PS.-ASCONIUS) : n. 99

ATHÉNÉE : n. 46

AUGUSTIN : n. 145

AULU-GELLE : n. 100

AUSONE : n. 101

AVANZI, GIROLAMO : n. 126

BARBARO, ERMOLAO : n. 178

BASILE DE CÉSARÉE : n. 62, 68, 146

BÉROALDE (L'ANCIEN), PHILIPPE : n. 124, 126, 179

BESSARION: n. 180 
BIBLE: n. 162-170, 174, 177

BIONDO, FLAVIO: n. 181

BOÈCE : n. 102

BOÈCE (PS.-) : n. 182

BONIFACE VIII: n. 184

BONISOLI, OGNIBENE (LEONICENO): n. 138

BUSSI, GIOVANNI ANDREA: n. 125

CALDERINI, DOMIZIO : n. 183, n. 29

CARMEN FAVINI : n. 14

CASSIODORE : voir « Historia ecclesiastica tripertita »

CATON : n. 103

CATULLE : n. 27

CEBETIS TABULA : n. 47

CELSE : n. 104

CÉSAR et PS.-CÉSAR : n. 105

CHION (PS.-): n. 68

CHEROBOSCUS, GEORGES: . 9

CICÉRON : n. 106, 190, 191

CICERON (PS.-) : n. 131

CLÉONIDÈS : n. 143

CODINUS (PS.-) : n. 200

COLUMELLE : n. 107

CORNÉLIUS NEPOS : n. 108

CORNUTUS, LUCIUS ANNÆUS : n. 20

CRATÈS DE MALLOS (PS.-) : n. 68

CURTIUS : voir « Quinte Curce »

CYPRIEN : n. 147

CYRILLE D'ALEXANDRIE : n. 148

DECEMBRIO, PIER CANDIDO : n. 39

DÉMÉTRIUS CHALCONDYLE : n. 4

DÉMÉTRIUS DE PHALÈRE : n. 69

DÉMOSTHÈNE (Ps.-) : n. 68

DÉMOSTHÈNE : n. 48, 49, 88, 94

DENYS D'HALICARNASSE : n. 69 
DENYS L'ARÉOPAGITE : voir « Pseudo-Denys »

DÉCRÉTALES : n. 184

DIGESTE : n. 109

DINARQUE : n. 83

DIODORE DE SICILE : n. 50

DIOGÈNE DE SINOPE (PS.-) : n. 68

DIOGÈNE LAËRCE : n. 40, 51

DIOGÉNIEN : n. 52

DIOMÈDE : n. 13

DION CHRYSOSTOME : n. 22

DIOSCORIDE (ET PS.-DIOSCORIDE) : n. 53

DONAT : n. 110

DONATUS AUCTUS : n. 111

ÉLIEN : n. 54, 193

ÉRASME : n. 146, 170, 185

ESCHINE (PS.-) : n. 67, 68

ESCHINE : n. 49, 83

ÉSOPE : n. 20

ÉTIENNE DE BYZANCE : n. 55

ETYMOLOGICUM MAGNUM : n. 2

EUCLIDE : n. 56

EURIPIDE : n. 21

EURIPIDE (PS.-) : n. 68

EUSÈBE DE CÉSARÉE : n. 149, 150, 159

EUSTATHE : n. 186

EUTROPE : n. 112

FAVINUS : voir « Remius Favinus »

FAZIO, BARTOLOMEO : n. 44

FESTUS / PAUL DIACRE : n. 113

FILETICO, MARTINO : n. 187

FIRMICUS MATERNUS : n. 114

FLAVIUS JOSÈPHE : n. 57

FLORUS : n. 115

FRONTIN : n. 143 
GALIEN : n. 40, 58, 59

GAZA, THÉODORE : n. 188-193

GENNADE : voir « Scholarios, Georges »

GEORGES LE MOINE : voir « Hamartolos, Georges »

GLOSE (Bible): n. 177

GLOSE (Corpus Iuris civ.): n. 109

GORGIAS: $n .83$

GRATIEN : n. 194

GRÉGOIRE DE CORINTHE : n. 4, 8

GRÉGOIRE DE NAZIANZE : n. 62, 146, 151, 152

GRÉGOIRE DE NYSSE : n. 153

GRÉGOIRE X : n. 184

HAMARTOLOS, GEORGES : n. 62, 200

HARPOCRATION : n. 5

HÉRACLITE (Ps.-) : n. 68

HERMOGÈNE : n. 69

HERMONYME, GEORGES : n. 3, 7, 9, 11, 49, 51, 52, 62, 67, 75, 76, 78, 84, 94, 162, 164-169, 190, 200

HÉRODE ATTIQUE : n. 83

HÉRODIEN : n. 60

HÉRODOTE (PS.-) : n. 22

HÉRODOTE : n. 61, n. 204

HÉSIODE : n. 23

HÉSYCHIUS : n. 6

HIÉROCLÈS : n. 62

HILAIRE DE POITIERS : n. 154

HIPPIATRICA : n. 195

HIPPOCRATE : n. 68

HISTOIRE AUGUSTE : n. 116

HISTORIA ECCLESIASTICA TRIPERTITA : n. 155

HOMÈRE : n. 22

HORACE : n. 28

HORAPOLLON : n. 20

INSTITUTES : n. 109 
ISÉE : n. 83

ISIDORE DE SÉVILLE : n. 196

ISOCRATE : n. 83

JEAN CHRYSOSTOME : n. 62, 156, 157

JEAN DAMASCÈNE : n. 158

JEAN PHILOPON : n. 40, 63

JÉRÔME : n. 159, 177

JULIEN : n. 64

JUSTIN : n. 117

JUSTIN (PS.-) : n. 160

JUSTINIEN : n. 109

JUVÉNAL : n. 29

LACTANCE : n. 161

LANDINO, CRISTOFORO : n. 197

LASCARIS, JANUS : n. 22, 41, 76, 79, 97

LESBONAX : $\mathrm{n} .83$

LETTRES D'ANACHARSIS : n. 68

LIBANIOS : n. $48,65,68$

LIBANIOS (PS.-) : n. 67

LIRANUS : voir « Nicolas de Lyre », n. 177

LUCAIN : n. 30

LUCIEN : n. 66, n. 94

LUCRÈCE : n. 31

LYSIAS : n. 83

MACROBE : n. 118

MAGENTINOS, LÉON : n. 198

MAGISTER, THOMAS: n. 7, 9

MARTIAL: $\mathrm{n} .32$

MARTIANUS CAPELLA : n. 119

MERULA, GIORGIO : n. 29

MOSCHOPULUS, MANUEL : n. 3, 4, 7, 9

NICOLAS DE LYRE : n. 177

NONIUS MARCELLUS : n. 15

NOUVEAU TESTAMENT : n. 162-170, 177 
CECUMENIUS : n. 164

ORIGÈNE : n. 171 ; voir aussi 173

OROSE : n. 120

OVIDE : n. 33

PANDECTES (DIGESTE DE JUSTINIEN) : n. 109

PAUL D’ÉGINE : n. 70, 71

PAUL DIACRE : n. 113

PAUSANIAS : n. 72

PEROTTI, NICCOLÒ : n. 199

PERSE : n. 34

PHALARIDE (PS.-) : n. 68

PHILON : n. 40

PHILOSTRATE : n. $67,68,73,74$

PHOCYLIDE (PS.-) : n. 75

PLANUDE, MAXIME : n. 94

PLATON : n. 68, 76, 77

PLATON (PS.-) : n. 76

PLAUTE : n. 35

PLÉTHON, GEORGES GÉMISTE : n. 200

PLINE L'ANCIEN : n. 121-125

PLINE LE JEUNE : n. 126

PLUTARQUE : n. 48, 78-81

PLUTARQUE (PS.-) : n. 22, 78, 79

POLITIEN, ANGE : n. 140, 143, 201

POLLUX : n. 10

POLLUX (PS.-) : n. 11

POLYBE : n. 85, 97

POMPONIUS MELA : n. 127

PORPHYRE : n. 40,82

PRISCIEN : n. 14

PROCLUS : n. 86

PSELLOS, MICHEL : n. 198

PSEUDO-DENYS L'ARÉOPAGITE : n. 172

PTOLÉMÉE : n. 87 
QUINTE CURCE : n. 128

QUINTILIEN : n. 129

RABAN MAUR : n. 202

REMIUS FAVINUS : n. 14

RHETORICA AD HERENNIUM : n. 200

RUFIN : n. 173

SABELLICO, MARCANTONIO : n. 179

SALLUSTE : n. 130

SALLUSTE (PS.-) : n. 131

SCHOLARIOS, GEORGES : n. 203

SCHOLIES À ARISTOPHANE : n. 19

SCHOLIES À DÉMOSTHÈNE : n. 88

SCHOLIES À EURIPIDE : n. 21

SCHOLIES À L'ILIADE : n. 22

SCHOLIES À L'ODYSSÉE : n. 22, 89

SCHOLIES À SOPHOCLE : n. 24

SCHOLIES AU PS.-DENYS : n. 172

SÉNÈQUE : n. 132

SEPTANTE : n. 174

SERVIUS : n. 133

SOPATER : n. 69

SOPHOCLE : n. 24

SOUDA [SUIDAS] : n. 12

STRABON : n. 90, 91

SUÉTONE : n. 134

SYNESIOS : n. 68

TACITE : n. 135

TÉRENCE : n. 36

TERTULLIEN : n. 175

THEMISTIUS : $\mathrm{n} .92$

THÉOCRITE : n. 25

THÉODORET : 176

THÉOGNIS : n. 26

THÉOPHRASTE : n. 40, 93 
THÉOPHYLACTE SIMOCATTA : n. 67, 68

THOMAS MAGISTER : voir « Magister, Thomas »

THUCYDIDE : n. 94

TITE-LIVE : n. 136

TROGUE POMPÉE : voir « Justin »

UGUCCIONE DE PISE : n. 16

VALÈRE MAXIME : n. 137

VALERIUS PROBUS : n. 17

VALLA, LAURENT : n. 130, 204, 205

VALTURIO, ROBERTO : n. 206

VARRON : n. 139-141

VARTHEMA, LUDOVICO DI : n. 207

VÉGÈCE : n. 142

VIE DE VIRGILE : voir « Donatus auctus »

VIRGILE : n. 37

VITRUVE : n. 143

VULGATE : n. 177

XÉNOPHON : n. 67, 95-97

\section{NOTES}

1. Voir Louis Delaruelle, Guillaume Budé. Les origines, les débuts, les idées maîtresses [1907], réimpr. Genève, Slatkine, 2012 ; Marie-Madeleine de La Garanderie, Christianisme et lettres profanes. Essai sur l'humanisme français (1515-1535) et sur la pensée de Guillaume Budé, Paris, H. Champion, 1995 ; ead. , Guillaume Budé, philosophe de la culture, Paris, Classiques Garnier, 2010 (avec la bibliographie antérieure à 2010) ; Gilbert Gadoffre, La Révolution culturelle dans la France des humanistes. Guillaume Budé et François I ${ }^{\text {er }}$, Genève, Droz, 1997.

2. Voir Jean-François Maillard et al., « Guillaume Budé », dans La France des Humanistes. Hellénistes I , Turnhout, Brepols, 1999, p. 93-96 ; Jean-Marc Chatelain, «Le Voyage de Varthema annoté par Guillaume Budé », Revue de la BnF, juin 1999, p. 67-71; Henri Omont, « Notice sur les collections de manuscrits de Jean et Guillaume Budé », Bulletin de la Société de l'histoire de Paris et de l'île-de-France, 1885, p. 100-113, et la bibliographie donnée infra.

3. Voir Luigi-Alberto Sanchi, Les Commentaires de la langue grecque. L'œuvre, ses sources, sa préparation, Genève, Droz, 2006; Guillaume Budé, De Asse et partibus eius / L'As et ses parties, éd. Luigi-Alberto Sanchi, t. I : Livres I-III, Genève, Droz, 2018 (avec une bibliographie spécifique). Sauf exception, la référence aux œuvres latines de Budé renvoie aux Budœi Omnia opera, éd. Celio Secondo Curione, Bâle, J. Froben, 1556, t. I-IV.

4. Budé, Opera, op. cit., t. III. Rappelons que l'on cite par ce titre une œuvre en deux livraisons : celle de 1508 (Paris, J. Bade), intitulée Annotations aux vingt-quatre [premiers] livres des Pandectes ( Annotationes in XXIV Pandectarum libros - ces livres forment par ailleurs la partie du Digeste appelée au Moyen Âge Digestum Vetus), puis le complément donné ibid. en 1526, Annotationes 
posteriores (Annotationes reliquae dans les Opera de 1556). En attendant une édition moderne, voir la description qu'en donne Delaruelle, Guillaume Budé, op. cit., p. 93-129; pour une liste provisoire d'auteurs cités, issue de sondages, voir Budé, De Asse, éd. Sanchi citée, p. XV, note 16.

5. Collection particulière, Genève, pour un total d'environ 1800 feuillets ; une reproduction est conservée à Paris, CNRS-IRHT. Voir Delaruelle, Guillaume Budé, op. cit., p. 247-277; Sanchi, Les Commentaires, op. cit., p. 142-146 et reproductions p. 311-316.

6. Omnia opera, op.cit., t. I, p. 362 . Voir Monique-Cécile Garand, «Les copistes de Jean Budé (1430-1502) », Bulletin de l'IRHT, 1967-1968, 15, p. 293-331 et Omont, « Notice sur les collections », cité.

7. Voir Jean-François Maillard et al., «Georges Hermonyme de Sparte ", dans La France des Humanistes. Hellénistes II, Turnhout, Brepols, 2010, p. 1-214; il est implicitement renvoyé à cet ouvrage de référence et à la bibliographie qu'il réunit lorsqu'on précise, ci-après, qu'un ms. a été copié par Hermonyme.

8. Voir Sanchi, Les Commentaires, op. cit., p. 125-130.

9. Voir ibid., p. 43-48.

10. Voir Budé, De Asse, éd. citée, p. XVIII-XXVIII.

11. S'agissant des œuvres de poésie, il y a lieu de distinguer les extraits convoqués par Budé pour des raisons d'étude (notamment de Plaute, Horace, Juvénal, Perse, Martial), d'un côté, et, de l'autre, les citations, tirées surtout de la poésie épique, qui ont pour but de souligner certaines idées dans les digressions politico-culturelles. Bien qu'il n'ait pas écrit en vers, Ésope est placé dans la section III.

12. Autres abréviations employées ici : prob(ablement), éd(ition), $\mathrm{E}($ dition)P(rinceps), $\mathrm{m}(\mathrm{anu}) \mathrm{s}$ (crit), m(anu)s(crit)s, trad(uction) lat(ine).

13. La pagination du De Asse à laquelle nous nous référons est celle de l'éd. Lyon, S. Gryphe, 1550-1551, in-8 ${ }^{\circ}$.

14. Pour des renseignements complémentaires, voir Sanchi, Les Commentaires, op. cit., p. 237-259 (répertoire des auteurs grecs cités) et p. 261-268 (index des auteurs grecs et latins cités), et Budé, De Asse, éd. citée, p. XCI-CXLVI (table des auteurs cités).

15. Voir ibid., p. 68 et 158-159.

16. On trouve cette même grammaire de Moschopulus dans un ms. copié par Hermonyme et relié aux armes de la famille Budé (Wien, ÖnB, Phil. gr. 92) : voir Maillard et al., Hellénistes II, op. cit., p. 21-22; or le filigrane datant de 1501 ne s'accorde pas avec l'époque de l'apprentissage du grec par Guillaume, le manuscrit aura pu être commissionné par un autre membre de la famille. Signalons aussi, au passage, le ms. Berne, Burgerbibl., 629 ([M. Planude], Vie d'Ésope ; M. Planude, trad. grecque des Disticha Catonis), initialement offert à Budé et dont les armes ont été grattées en vue du réemploi : voir ibid., p. 167-169.

17. Voir Sanchi, Les Commentaires, op. cit., p. 158 (et 67-68).

18. Voir Joseph Quesnel, Catalogus Bibliothecee Thuance, t. II, Hambourg, C. Liebezeit, 1704, p. 231.

19. Par exemple [Opera], Venise, B. Locatelli, 1497 (exemplaire consulté : Paris, BnF, Rés. X. 570), ou [Opera], Venise, G. Arrivabene, 1488 (exemplaire consulté : BnF, Rés. X. 592) ; voir Budé, De Asse , éd. citée, p. XXIX-XXXI.

20. Voir l'éd. Theodor Mommsen dans Heinrich Keil, Grammatici Latini, t. IV, Leipzig, 1864.

21. Voir l'ouvrage récent de Patrick Morantin, Lire Homère à la Renaissance. Philologie humaniste et tradition grecque, Genève, Droz, 2017, en particulier p.195-213, où est aussi reprise la bibliographie sur la question.

22. Commentateurs mentionnés dans $D A$; voir, à titre d'exemple, Iuuenalis cum tribus commentariis, videlicet Domitii Calderini, Georgii Merule, necnon Georgii Valloe, Venise, S. Bevilacqua, 1497 ; Satyrce cum commentariis Domitii Calderini, Venise, B. de Zani, 1487 (exemplaire numérisé).

23. Voir Budé, De Asse, éd. citée, p. XXVI-XXVII. 
24. Les annotations de Budé aux t. II-V, 1495-1498, aujourd'hui perdus, sont attestées par le Catalogue de la Bibliothèque du roi de Pierre et Jacques Dupuy (1647), sous les n. 456 (t. III), 480 (t. V), 481 (t. II), 821 et 900 (t. IV en deux volumes) ; voir Sanchi, Les Commentaires, p. 161, pour la liste complète des contenus de cette édition.

25. Rappelons que Budé est l'auteur d'une traduction latine de ce traité, à laquelle se joint celle du De mundo du Ps.-Aristote (Paris, J. Bade, [1526]).

26. Voir Sanchi, Les Commentaires, p. 127.

27. Voir ibid., p. 127.

28. Voir Budé, De Asse, éd. citée p. XL (cf. XIX, note 31).

29. Voir Sanchi, Les Commentaires, p. 129-130.

30. Voir Maillard et al., « G. Budé », dans Hellénistes I, op. cit., p. 66, 68, 69.

31. Budé semble reprendre le titre abrégé de l'Aldine; les manuscrits portent plutôt 'EK $\tau \tilde{\omega} v$

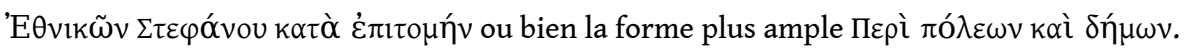

32. DA, p. 475, à propos d'un passage annoncé comme tiré des «Antiquités, livre VIII », mais qui correspondrait plutôt au Contre Apion; lorsqu'il ne cite Flavius Josèphe qu'en latin - c'est le cas pour la Guerre des Juifs - Budé est apparemment l'auteur de la traduction et, d'ailleurs, p. 496, au sujet du Contre Apion, il dénonce la mauvaise qualité de la traduction latine attribuée à Rufin ; voir aussi p. 610, à propos d'Ant. J., III, 321.

33. Voir Sanchi, Les Commentaires, op. cit., p. 128 et note 20.

34. Voir Luigi-Alberto Sanchi, « Guillaume Budé lecteur d'Hérodote : langue, idées, recherches », Anabases, 2010, p. 9-18.

35. Le recueil d'Alde ne comprend pas les lettres de Grégoire de Nazianze.

36. Pour une description détaillée des contenus, voir Sanchi, Les Commentares, op. cit., p. 160.

37. Hypothèse à vérifier après un dénombrement entier des citations platoniciennes des Ann. de 1508, antérieures à la princeps; un premier sondage permet de cerner des citations du Minos (Budé, Opera, t. III, p. 104-105) et une des Lois (ibid., p. 149), sans doute extraites du ms. d'Hermonyme.

38. Opinions de philosophes, Fortune des Romains, Fortune d'Alexandre, Apophtegmes des rois et des empereurs, Apophtegmes laconiens, Banquet, Maxime cum princ., De sera num., De laude ips., Fortune, De tuenda san., Parallèles grecs et romains, Ætia Rom., Ætia Gr., Quomodo adulator, De cohib. ira, De tranqu., De cupiditate div., De recta rat. aud., Curiosité, Consol. à Apoll. Rappelons que Budé est l'auteur de trad. lat. de plusieurs opuscules de ce corpus : De tranq., Fort. Rom., Fort. Alex. (Paris, J. Bade, 1505, avec une lettre de St. Basile) Ps.-Plut., Opin. philos. (Paris, J. Bade, 1505/6)

39. Voir Sanchi, Les Commentaires, op. cit., p. 126.

40. Voir, à titre d'exemple, Vitæe, t. I-II, Venise, Bartolomeo Zani, 1496 (exemplaire consulté : BnF, Rés. J. 820).

41. Voir Morantin, Lire Homère, op. cit., p. 214-215.

42. La princeps de Strabon (Venise, A. Torresano) date de «1516»; or le De Asse comporte dès la première éd., parue en mars 1515 , plusieurs citations grecques de cet auteur.

43. Voir V. Juřen, "Ex Bibliotheca Gulielmi Budæi ", Revue d'histoire des textes, 1976, vol.6, p. 341-343; les carnets autographes de Budé conservés à Genève incluent des extraits tirés de Celse : voir Delaruelle, Guillaume Budé, op. cit., p. 252-259 (carnets numérotés II, III et IV).

44. Liste d'écrits cités dans le DA: Brutus, Caton l'Ancien (voir aussi la traduction en grec de Théodore Gaza), Contre Pison, Correspondance, De la divination, De la Nature des dieux, De l'Orateur, Des Termes extrêmes des biens et des maux, Les Devoirs, Les Paradoxes des Stoïciens, L'Orateur, Philippiques, Pour Balbus, Pour Coelius, Pour Cécina, Pour Cluentius, Pour Flaccus, Pour le poète Archias, Pour Q. Roscius le Comédien, Pour P. Quinctius, Pour Rabirius, Sur la loi agraire (Contre Rullus), Sur les pouvoirs de Pompée (Pour la loi de Manilius), Sur sa maison, Timée (De Uniuersitate), Tusculanes, Verrines (avec le Discours contre Q. Cacilius, dit « La Divination»). 
45. Voir DA, p. 125 (ms. des Verrines), p. 237 (le «volumen antiquum Orationum Ciceronis » que Budé a pu consulter ne contient pas le Pro Rosc. Com., ni deux autres); p. 287, l'auteur précise qu'il n'a pas pu trouver le Pro Rabir. Posth. dans un « vetustus liber Orationum Ciceronis », sans doute le même volume; voir, pour l'hypothèse de Saint-Victor, Henri de la Ville de Mirmont, Jean Humbert, Discours, t. I/1, CUF [1921] 1960, p. VII : référence à Cicéron, Orationes, Paris, J. Bade, 1522 (qu'ils datent «1527»), épître de Bade à Dreux Budé, extrait reproduit dans Maillard et al., "Jacques Thouzat ou Tusan », Hellénistes II, op. cit., p. 416-417, où toutefois il n'est évoqué qu'un « livre de Budé ".

46. Voir Budé, De Asse, éd. citée, p. XXXIII.

47. Voir, à titre d'exemple, Seruii Commentarii in Virgilium, éd. Battista Guarino, Venise, C. Baldafer, 1471, f. [1]-[7] ; voir en outre l'édition moderne de Fabio Stok, «Vita quæ Donati Aucti dicitur », dans Vitee Virgiliance antiquee, éd. Giorgio Brugnoli, Fabio Stok, Rome, Istituto Poligrafico e Zecca dello Stato, 1997, p. 71-135.

48. Budé corrige dans le DA, p. 636, une leçon «des imprimés » en leur opposant la leçon des " anciens » (" ut in impressis legimus contra fidem antiquorum »).

49. Pour cette source du texte plinien et pour les quatre suivantes, voir Budé, De Asse, éd. citée, p. XXXIII-XXXV, et la bibliographie fournie ibid.; le premier ms. a déjà été identifié par Louis Delaruelle, les autres identifications émanent à la fois de ce que Budé indique lui-même dans les Ann. et surtout dans le $D A$ ainsi que de la collation des nombreuses variantes typiques citées dans le $D A$.

50. Voir DA, p. 141 : "sic enim in membranaceo legimus", et p. 417, 418 et 428, où il oppose ce ms. aux « codices impressi ». On trouve un éloge ibid, p. 418 («Curtii, auctoris tersi atque elegantis »).

51. Voir DA, p. 272 : « Fortasse [...] apud Suetonium legitur. Mihi enim antiquum videre non contigit. »

52. Voir DA, p. 611 (« in aliis exemplaribus »).

53. Voir DA, p. 330 ; voir aussi, à titre d'exemple, Opus Valerii Maximi cum Omniboni Leoniceni interpretatione, Venise, G. da Forlì et socii, 1482 au colophon (exemplaire consulté : Paris, BnF, Rés. Z. 185).

54. Il fait état de variantes « ex vetusto » dans DA, p. 603-604.

55. Voir DA, p. 227 ( = III, 6, 6: Albutius a été corrigé par Politien) et p. 228 ( = III, 2, 17 : omission d' agro, comme dans l'éd. princeps).

56. Voir la mention figurant sur la page de titre de l'édition de Paris, C. Wechel, 1532 (« De Re militari libri quatuor » etc.) : " collata sunt omnia ad antiquos codices, maxime Budœi »

57. Voir Sanchi, Les Commentaires, p. 241-242.

58. Voir DA, p. 473 : « Grocum exemplar non vidi».

59. L'exemplaire de la BnF est le t. IV, mais les t. I-III sont attestés dans le catalogue de la bibliothèque de Thou : voir Chatelain, «Le Voyage ", art. cité, p. 70-71, $\mathrm{n}^{\text {os }} 13$ et $13^{\text {bis }}$.

60. Annotations aux Pandectes : voir Opera, t. III, p. 56d.

61. L'évangéliaire grec consulté par Budé semble correspondre à l'item «B 19 » du catalogue de 1514, car il est le seul possédé à ce moment : voir G. Ouy, Le Catalogue de la bibliothèque de l'abbaye de Saint-Victor de Paris de Claude de Grand-rue, 1514, Paris, Cnrs, 1983, p. 11 ; Id., Les manuscrits de l'Abbaye de Saint-Victor. Catalogue établi sur la base du répertoire de Claude de Grand-rue (1514), Turnhout, Brepols, 1999, t. II, p. 21 ; voir aussi Delaruelle, Guillaume Budé, p. 106 et n. 3, 118 et n. 3 (cf. p. 105, n. 2).

62. Voir Maillard et al., Hellénistes II, op. cit., p. 61 et note 82.

63. Voir Luigi-Alberto Sanchi, « Guillaume Budé et la critique érasmienne du Nouveau Testament en latin ", sous presse.

64. Budé attribue la trad. lat. à Jérôme.

65. Pour les commentaires, voir les éd. d'auteurs classiques cités supra ; certains auteurs figurent dans la section I. 
66. Voir, à titre d'exemple, Commentationes conditce a Philippo Beroaldo adiecta paraphrastica M. A. Sabellici interpretatione in Suetonium Tranquillum, Paris, L. Hornkem, G. Hittorp, 1512 (exemplaire consulté : Paris, BnF, Rés. G-J-4).

67. Voir, à titre d'exemple, Commentarii in Martialem, Venise, J. de Colonia Agrippinensi, 1474 (exemplaire consulté : Paris, Arsenal, Fol-BL-673)

68. Dans nombre de cas, Budé ne cite pas explicitement le nom d'Érasme, en particulier lorsqu'il cite un proverbe sans doute tiré des Adages, mais qu'il a pu également puiser à la source antique ; le développement anti-cicéronien inséré dans les Comm. en 1529 est à lier à la publication du Ciceronianus, controversée à Paris dans les milieux humanistes.

69. Pour l'utilisation d'Eustathe comme source parallèle aux scholies homériques, voir Morantin, Lire Homère, op. cit., passim.

70. Voir, à titre d'exemple, Hoc in volumine... Ciceronis Epistolarum familiarium libri XVI... Martini Philetici in quasdam epistolas ellectas [sic] commentarii..., Lyon, J. de Vingle, 1496 (exemplaire numérisé).

71. Voir, à titre d'exemple, In hoc volumine... Theodori... De mensibus opusculum..., Venise, Alde, 1495, f. [89]-[106].

72. Ce ms. a appartenu à la famille de Mesmes, comme plusieurs autres de la bibliothèque de Budé : voir H. Omont, "Collections de Jean et Guillaume Budé», art. cité, p. 112 ; voir aussi Hellénistes II, $\mathrm{n}^{\text {os }} 39,90,95$.

73. Voir, à titre d'exemple, Aristotelis De natura animalium..., Lyon, B. de Gabiano, 1505.

74. Texte de vétérinaire du IX ${ }^{\mathrm{e}}$ s. ; l'éd. princeps date de 1537 : Veterinarice medicince libri II..., Bâle, S. Grynæus.

75. Budé reprend la forme erronée «Margent- » dans les Commentaires : Budé, Opera, op. cit., t. IV, col. 246, 47.

76. Voir Luigi-Alberto Sanchi, «Per la ricezione di Poliziano in Francia : spunti dall'opera erudita di Guillaume Budé », Archivum Mentis, 2014, p. 233-246.

77. Voir Sanchi, Les Commentaires, op. cit., p. 246.

78. Voir ibid., p. 72-73;110-111.

79. On trouvera en outre les noms des intermédiaires humanistes ou copistes cités dans certaines entrées. Les numéros renvoient aux items de la bibliothèque ci-dessus.

\section{RÉSUMÉS}

Avec le dépouillement du contenu érudit des cinq livres De Asse et partibus eius (1515; septième éd., 1541), qui s'ajoutent à celui des quelque vingt mille citations formant les Commentarii linguce Graeca (1529; éd. définitive, 1548), il est possible d'enrichir sensiblement la liste de livres de la bibliothèque intellectuelle de Guillaume Budé (1468-1540), dont une partie seulement est matériellement identifiée. Il en ressort un vaste tableau, certes à mettre à jour dès que le contenu des deux éditions d'Annotationes in Pandectas (1508 et 1526 ; éd. définitive, 1535) sera précisément connu, mais signalant déjà Budé comme un lecteur aussi vorace qu'encyclopédique, bon connaisseur des classiques mais surtout grand explorateur des textes ardus des grammairiens, des théologiens, des juristes, des agronomes et d'autres auteurs dits techniques.

Thanks to the counting of the contents of the five books De Asse and partibus eius (1515; seventh edition, 1541), which are added to some twenty-thousand quotations forming the Commentarii 
linguce Grcece (1529; final edition, 1548), it is now possible to substantially enrich the list of Guillaume Budé's (1468-1540) intellectual library, only a part of which is physically identified. It turns out to be a vast array, certainly to be better known later, when the contents of the two editions of Annotationes in Pandectas (1508 and 1526; final edition, 1535) will be fully studied, but already signaling Budé as a reader as voracious as encyclopedic, a good connoisseur of the classics, but above all a great explorer of the difficult texts of grammarians, theologians, jurists, agronomists and other authors, the so-called technici.

INDEX

Keywords : Budé (Guillaume), French Renaissance, classical scholarship, intellectual library, antiquarianism

Mots-clés : Budé (Guillaume), Renaissance française, philologie classique, bibliothèque intellectuelle, érudition

\section{AUTEUR}

\section{LUIGI-ALBERTO SANCHI}

Institut d'histoire du droit (Cnrs-IHD), Paris 